\title{
EFFECT OF CYLINDER ROUGHNESS ON STROUHAL NUMBER
}

\author{
V. Yanovych ${ }^{1,2}$, D. Duda ${ }^{1}$, V. Uruba ${ }^{1,2}$, P. Procházka ${ }^{2}$, P. Antoš ${ }^{2}$ \\ ${ }^{1}$ Department of Power System Engineering, Faculty of Mechanical Engineering, \\ University of West Bohemia, Univerzitní 8, Pilsen 30100 , Czech Republic \\ ${ }^{2}$ Czech Academy of Sciences, Institute of Thermomechanics, Dolejškova 5, Prague 182 \\ 00, Czech Republic
}

\begin{abstract}
The main goal of this paper is to establish a better understanding of the relationship between a Strouhal number and surface roughness. Hot-wire anemometry was used to evaluations the characteristics of turbulent flow behind circular cylinders with different relative roughness $0 \%$ (smooth surface) $0.83 \%, 1.67 \%, 3.33 \%$, and $6.67 \%$. At the experimental investigation, the Reynolds number based on the cylinder diameter was $5 \times$ $10^{3}<\operatorname{Re}_{\mathrm{d}}<2 \times 10^{4}$. The obtained data showed that the Strouhal number decreased with increasing roughness. While, the dissipation rate decreases, and the value of the Kolmogorov and Taylor microscales increases. Also, spectral analysis of streamwise velocity fluctuations allowed us to estimate the location of the vortex-shedding frequency which at growing roughness tends to reduce.
\end{abstract}

Keywords: roughened cylinder, Strouhal number, spectral distribution, turbulence structure, Hot-wire anemometry

\section{Introduction}

The flow behavior through the circular cylinder is one of the most common issues in fluid mechanics. That is due to its widespread occurrence in various engineering applications. Thus, for example, cylinders constructions are widely provided in heat exchange equipment, gas compressors or steam turbines, various turbomachines, and other aerodynamic system [1-4]. In most cases, the surface of the cylindrical bodies is smooth. But due to the influence of various operational factors, it gradually becomes rough over time.

Many researchers have vastly studied the effect of surface roughness on turbulence topology in recent decades. The study of roughness in turbulent flows was initiated by Hagen [5] and Darcy [6], who found that roughness affects the pressure drop by increasing the resistance and blocking effects [7]. In addition, many scientists experimentally confirmed that the roughness leads to a decrease in the Strouhal number and the vortex-shedding frequency [8-11]. Understanding the mechanism of these phenomena is important for the future design of cylindrical constructions and predicting their behavior in different applications.

In this work, the roughness effect on the universal Strouhal number of vortex frequencies and turbulent flow structure behind a circular cylinder was investigated. The obtained experimental results also show a clear relationship between the dissipation characteristics of the flow and the value of surface roughness.

\section{Experimental setup}

The experiments were performed in an open-type wind tunnel, which was developed in the Laboratory of Turbulent Shear Flows of the Institute of Thermomechanics of the Czech Academy of Sciences (IT CAS). The scheme of the experimental investigation is shown in (Fig. 1(a)). The operating flow velocities were $5,10,15$ and $20 \mathrm{~m} \cdot \mathrm{s}^{-1}$. It is should be noted that the special design of the tunnel allows generating flow with a low degree of turbulence $0.3 \%$. The internal dimensions of the test section were $250 \mathrm{~mm}$ (height), 250 (width) $\mathrm{mm}$ and $750 \mathrm{~mm}$ (length), respectively.

To assess the effect of roughness on the flow topology, we used cylinders whose surfaces had different roughness (see Fig. 1(b)). They were produced of $250 \mathrm{~mm}$ long aluminum tubes and covered with sand. The grain size of which determined the height of the surface roughness. Accordingly, to our investigations, the granularity was $0.125 \mathrm{~mm}, 0.25 \mathrm{~mm}, 0.5 \mathrm{~mm}$, and $1 \mathrm{~mm}$. One smooth cylinder was employed to provide a benchmark with the results of the rough cylinders. The diameter of this cylinder 
was $15 \mathrm{~mm}$. However, the diameters of the other cylinders increase slightly with increasing roughness. The fabricated bluff bodies were vertically placed in the middle of the test section, while their centers were located at a distance of $3 d$ from the inlet.

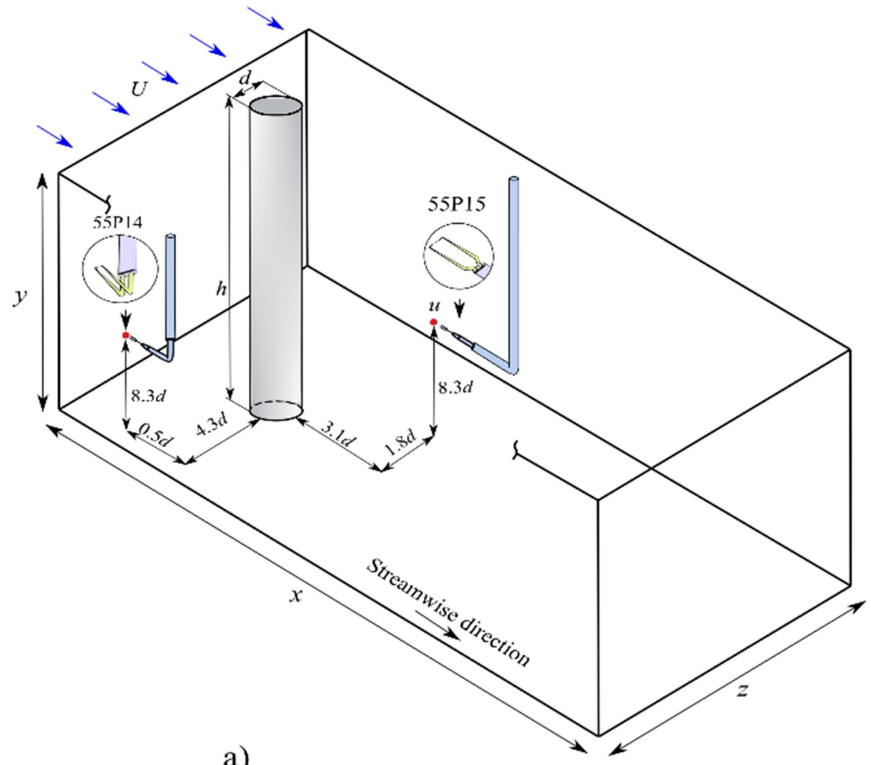

a)

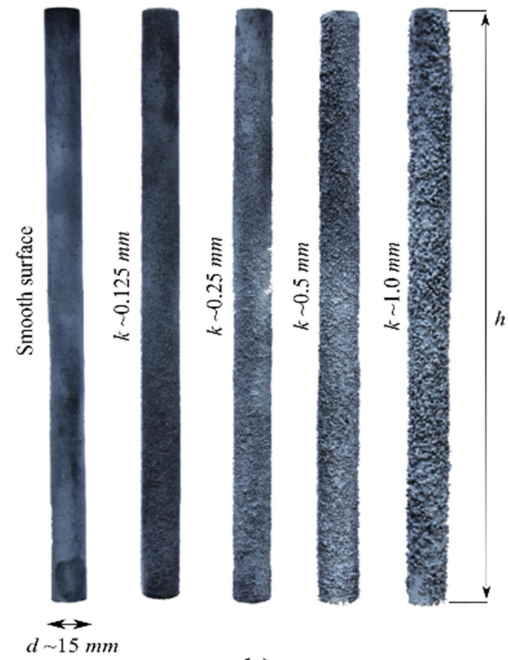

b)

Figure 1: a) Scheme of the experimental investigation. The arrows in blue indicate the direction of air flow. The red and blue dots are the locations of the Hot-wire probes for measuring reference $U$ and streamwise $u$ velocity, respectively. While $d$ and $h$ are the diameter and length of the cylinder, respectively. While $k$ is the height of the roughness. b) Experimental models of roughened cylinders.
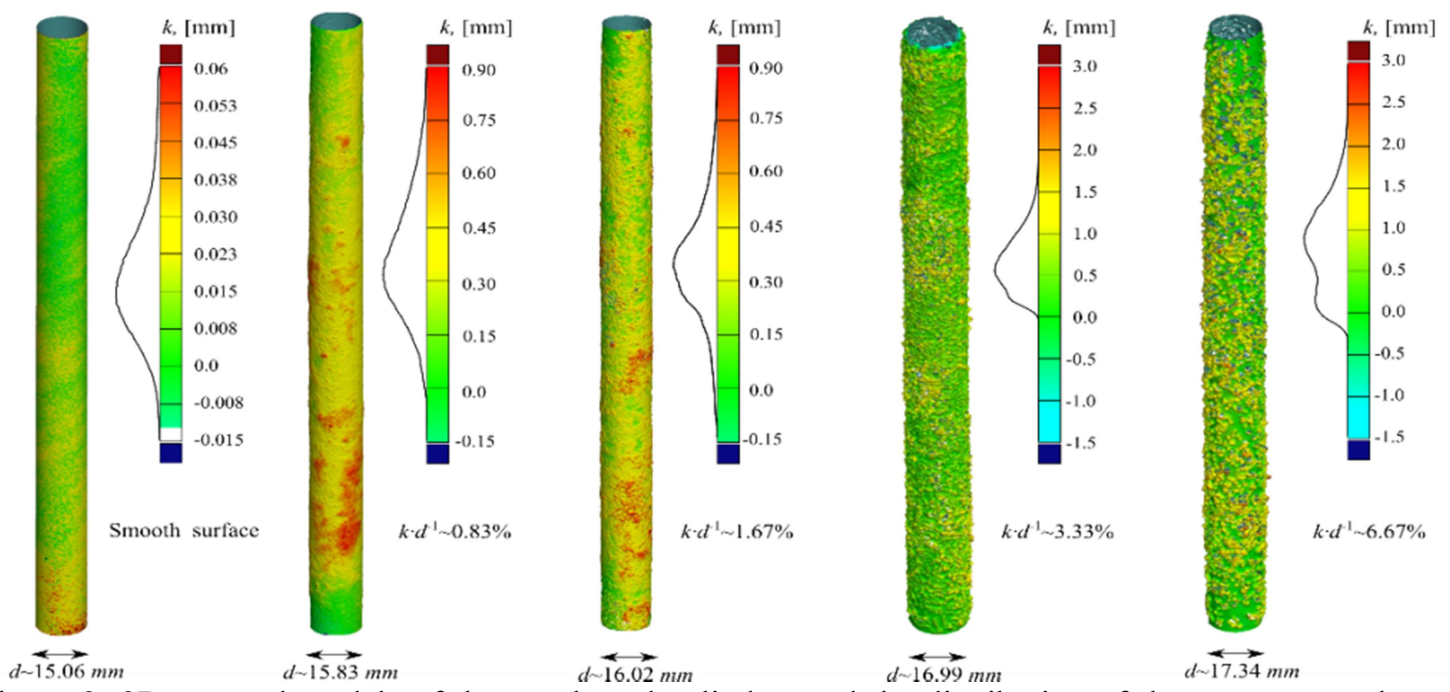

Figure 2: 3D scanned models of the roughened cylinders and the distribution of the average roughness heights. Where $k$ and $d$ are the average height of the roughness and the diameter of the cylinder, respectively. While $k \cdot d^{-1}$ reflects the relative roughness value.

Measurements were performed at two points in front and behind the cylinder. Moreover, they were located with an offset in the horizontal plane relative to the cylinder axis. Accordingly, the first point was used to measure the reference velocity $U$, while the second one to analyze the streamwise component $u$ of the flow. For these purposes, we used 55P14 and 55P15 hot-wire probes. The wire probes with tungsten platinum coating have a diameter of $5 \mu \mathrm{m}$ and an active length of $1.25 \mathrm{~mm}$. While the wire ends are gilded. The hot-wire signals for all experiments were sampled at $75 \mathrm{kHz}$. The filtering of the received signal was also applied. For the high and low pass filter the frequency was $10 \mathrm{~Hz}$, and $30 \mathrm{kHz}$ respectively. While, the point velocity measurement was $10 \mathrm{~s}$. Accordingly to methodologies [12], the 
uncertainty of the conversion process of a probe's electrical signal into velocity samples was about $\pm 0.5 \%$.

To accurately determine the actual geometry of the manufactured cylinders, we used the 3D noncontact optical scanning system ATOS Core. Measurement data from this system formed the foundation of three-dimensional quality control of the finished experimental bluff body. Based on them, we calculated the actual average height of roughness $k$ and the external diameters $d$ of the produced cylinders. Moreover, using these values, it is easy to find the characteristics of the relative roughness $k \cdot d^{-1}$ (see Fig. 2). The value of which is in the range from $0.83 \%$ to $6.6 \%$. Meanwhile, the external diameter of the cylinder increases from $15.06 \mathrm{~mm}$ (smooth cylinder) to $17.34 \mathrm{~mm}$. Thus, according to formula 1 , diameter-based Reynolds numbers will increase by $15 \%$. It should be noted that this trend is constant at all experimental velocities of the experiment. For example, at $U=20 \mathrm{~m} \cdot \mathrm{c}^{-1}$ for the smooth cylinder and for maximum roughened cylinder $\left(k \cdot d^{-1} \sim 6.6 \%\right)$, the $R e_{d} \sim 20 \cdot 10^{4}$ and $R e_{d} \sim 23 \cdot 10^{4}$, respectively.

$$
R e_{d}=(U d) \cdot v^{-1}
$$

where $U$ is the reference velocity, $d$ is the real cylinder diameter and $v$ is the kinematic viscosity of air.

Also, the data clearly show that the nature of the roughness location is not homogeneous. This feature significantly complicates the possibility of accurate repetition of experimental measurements.

\section{Results and discussion}

Strouhal suggested that the regularity of vortex shedding phenomenon is described in the term of a nondimensional number and can be represented in the form:

$$
S t=\left(f_{s} d\right) \cdot U^{-1}
$$

where $f_{s}$ is the vortex-shedding frequency, $d$ across-wind dimension of the bluff body band $U$ is the mean velocity of the uniform flow.

To determine the $f_{s}$, we analyzed the streamwise signals using the power spectral density. Which shows the pattern of distribution in the time domain of signal power over the different frequencies. Graphical interpretations of the obtained spectral are shown in Fig. 3.

As we can see, the resulting curves have clear peaks in some frequency ranges. They correspond to the vortex-shedding frequency. It should be noted that this value, regardless of the velocity, decreases by $16.5 \%$ with increasing relative roughness to $k \cdot d^{-1} \sim 6.6 \%$. This pattern can be caused not only by increasing the surface roughness but also by increasing the external diameter of the cylinder. Another interesting feature of the obtained distributions is the presence of the second harmonic in the longitudinal fluctuations of the flow. The amplitude of which decreases with increasing velocity.

In addition, the obtained data clearly show that the slope of the spectral distribution curve varies greatly depending on the roughness. That is especially noticeable at the low velocity. For example, at 5 $\mathrm{m} \cdot \mathrm{c}^{-1}$ the slope for a smooth cylinder is $f^{5}$. While for cylinder with relative roughness of $6.67 \%$, it is equal to $f^{5 / 3}$. In the second case, according to Kolmogorov's theory, this slope may indicate an isotropic homogeneous characteristic of turbulent flow. While in the first one, a steeper slope may indicate nondeveloped turbulence [13].

It should note that as the roughness increases, the intensity of turbulence growing also (Fig. 4 (a)). That is especially noticeable at $5 \mathrm{~m} \cdot \mathrm{c}^{-1}$. Where IT gradually increase depending on the surface roughness from $3.5 \%$ to $7.7 \%$. While at $U=20 \mathrm{~m} \cdot \mathrm{c}^{-1}$ in all cases except for a smooth cylinder, it is $6.5 \%$.

Finally, substituting the values of the vortex shedding frequency and the actual external diameter of the roughened cylinders into formula 1, the Strouhal number was determined. Graphic interpretation of the obtained data is shown in (Fig. 4 (b)).

As we can see, there is a clear tendency to reduce the Strouhal number with increasing value relative roughness. Thus, when increasing $k \cdot d^{-1}$ to $6.67 \%$ compared to a smooth cylinder, $S t$ decreases by $4.5 \%$. This pattern is approximately similar for all velocities modes. In addition, as we can see at a constant roughness, the change in velocity has no significant effect on the magnitude of $S t$.

To better understand the effect of roughness on the flow topology, we tried to investigate the development of the turbulence structure in the measuring point behind cylinders. For this purpose, we used a methodology that is well described in the papers $[14,15]$. The calculations results are shown in Fig. 5.

One of the main points of the study was the estimation of the dissipation rate of the flow depending on the surface roughness characteristics. This can be found using the second-order Eulerian longitudinal structure function. According to Kolmogorov's law at large Reynolds numbers, it can be found as:

where $C \sim 2.1$ is imperial coefficient.

$$
S^{2}(t)=C \cdot(\epsilon \cdot t)^{2 / 3}
$$




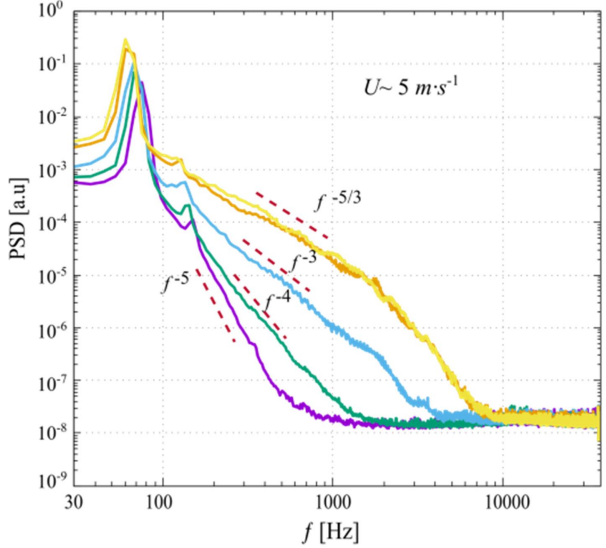

a)

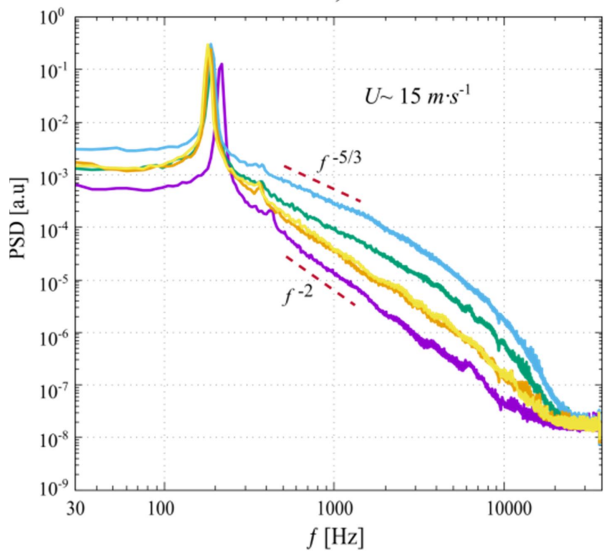

c)

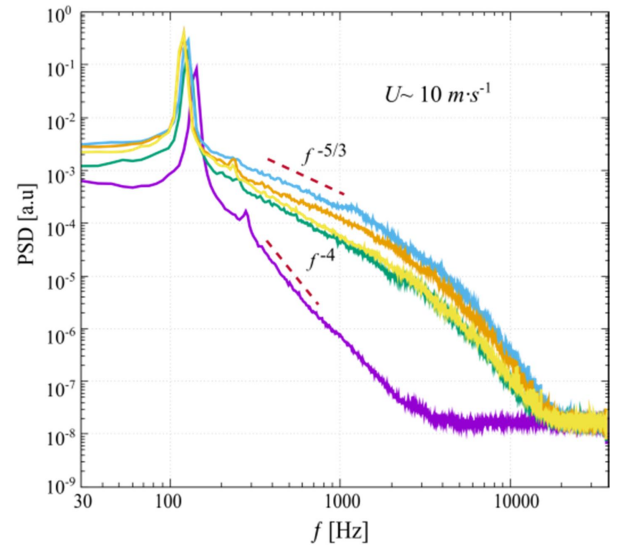

b)

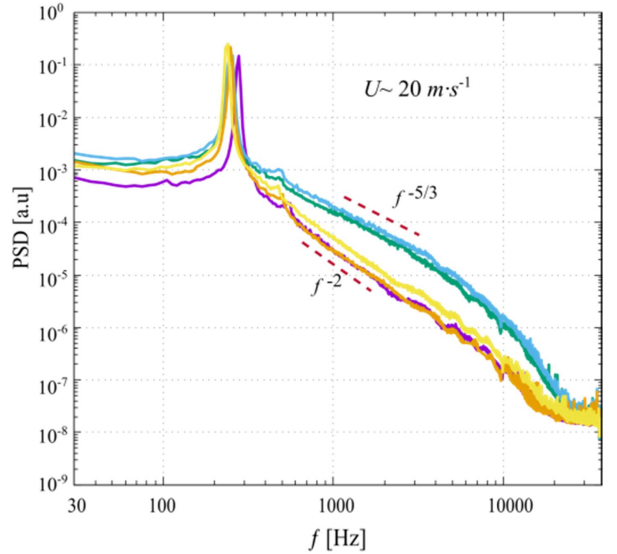

d)

$$
0-0.83 \%-1.67 \%-3.33 \%-6.67 \%
$$

Figure 3: Power spectral densities of streamwise velocity fluctuations depending on the relative roughness $k \cdot d^{-1}$ and reference velocity $U$. Purple, green, light blue, orange, and yellow colors correspond to the relative roughness of $0.83 \%, 1.67 \%, 3.33 \%$, and $6.67 \%$, respectively. While a), b), c) and d) show the spectral characteristics at 5,1015 and $20 \mathrm{~m} \cdot \mathrm{c}^{-1}$.

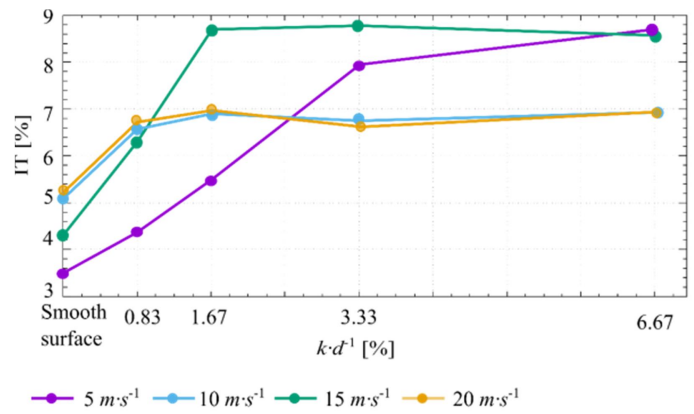

a)

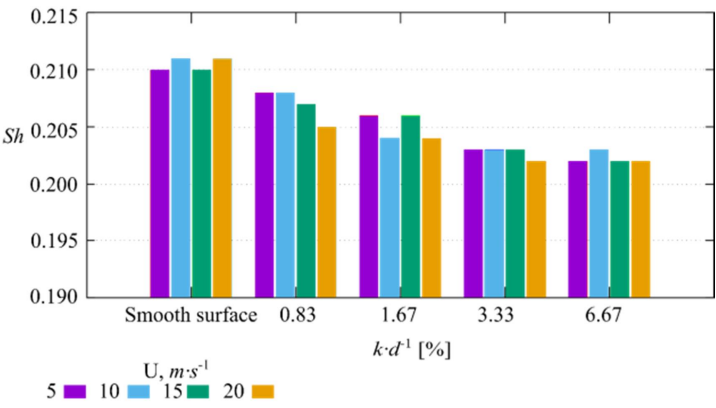

b)

Figure 4: Intensity of turbulence a) and Strouhal number St b) depending on the relative roughness $k \cdot d^{-1}$ and velocity $U$. Purple, light blue, green, and orange colors correspond to the different reference velocities $5,10,15$, and $20 \mathrm{~m} \cdot \mathrm{s}^{-1}$, respectively.

As we can see from Fig. 4(a), velocity has the greatest effect on dissipation rate. Thus, when increasing the flow velocity from $5 \mathrm{~m} \cdot \mathrm{s}^{-1}$ to $20 \mathrm{~m} \cdot \mathrm{s}^{-1}$, the value of $\epsilon$ grows proportionally almost four times. However, there is also a small effect of roughness. That is especially noticeable when the relative roughness increases up to $k \cdot d^{-1} \sim 1.67 \%$. Then at $10 \mathrm{~m} \cdot \mathrm{s}^{-1}$ and $15 \mathrm{~m} \cdot \mathrm{s}^{-1}$, the value of dissipation rate is almost stabilized regardless of roughness characteristics. While at $5 \mathrm{~m} \cdot \mathrm{s}^{-1}$ and $20 \mathrm{~m} \cdot \mathrm{s}^{-1}$ it continues to 
decrease slightly. In general, regardless of the velocity, with an increase in roughness up to $k \cdot d^{-1} \sim 6.67 \%$, the $\epsilon$ decreases by $13-16 \%$. Using the obtained values of $\epsilon$, we also calculated the dissipation scale $\eta$ from the relations 1 . This parameter characterizes the average size of the smallest flow vortices.

$$
\eta=\left(v^{3} \cdot \epsilon^{-1}\right)^{1 / 4}
$$

Graphical interpretation of the dissipation scale is shown in Fig. 4(b). This shows that the most influential factor on the $\eta$ is the flow velocity. In addition, also observed, increasing the granularity size of the surface leads to the growth of the smallest vortices which, according to Kolmogorov, plays the main role in the energy dissipation of turbulent flow. In our case, in the whole range of experimental velocities, with increasing relative roughness up to $k \cdot d^{-1} \sim 6.67 \%$, the value of dissipation scales increases approximately by $4 \%$.

Also, we found Taylor microscale $\lambda$ and the Reynolds number based on it $R_{\lambda}$, accordingly to relations:

$$
\begin{aligned}
& \lambda=\sigma_{u}\left(15 \cdot v \cdot \epsilon^{-1}\right)^{1 / 2}, \\
& R_{\lambda}=\left(\sigma_{u} \lambda\right) \cdot v^{-1},
\end{aligned}
$$

where $\sigma_{u}$ is standard deviation of streamwise velocity component $u$.

Analysis of the Taylor microscale distribution shows that its value changes significantly with increasing flow velocity, especially in the presence of roughness (see Fig. 4(c)). Thus, it can conclude that increasing the roughness leads to a reduction in the inertial range, where the viscosity of the turbulent flow does not affect the overall dynamics of the vortices. It should be noted that sharp differences for the Taylor microscale are observed in the velocity range from $10 \mathrm{~m} \cdot \mathrm{s}^{-1}$ to $20 \mathrm{~m} \cdot \mathrm{s}^{-1}$ up to $k \cdot d^{-1} \sim 1.67 \%$. After that, the value of $\lambda$ stabilizes. Whereas at $5 \mathrm{~m} \cdot \mathrm{s}^{-1}$, where it continues to grow gradually with increasing roughness. In general, at $10 \mathrm{~m} \cdot \mathrm{s}^{-1}$ to $20 \mathrm{~m} \cdot \mathrm{s}^{-1}$ and at maximum roughness, the value of the Taylor microscale increases around 1.5 times relative to a smooth surface.

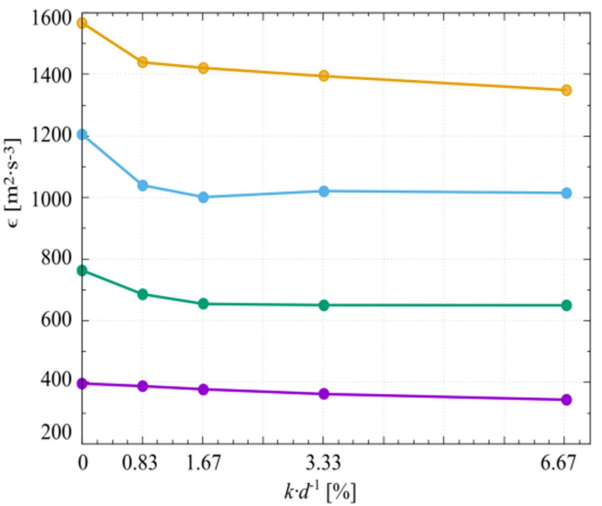

a)

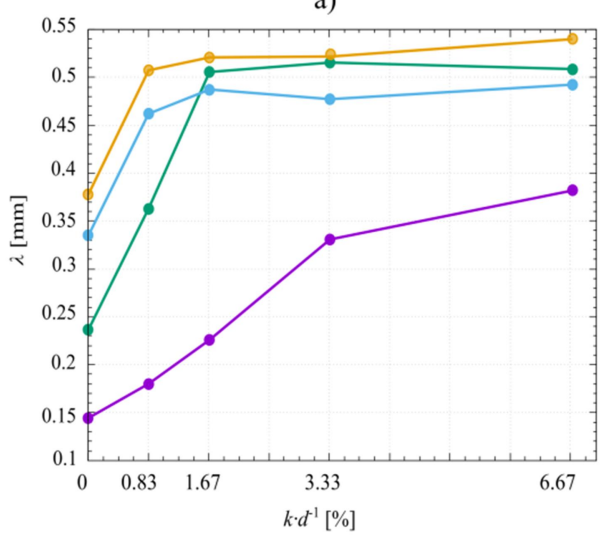

c)

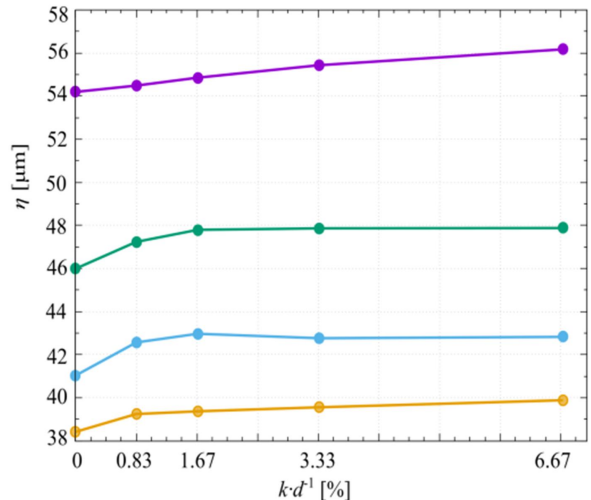

b)

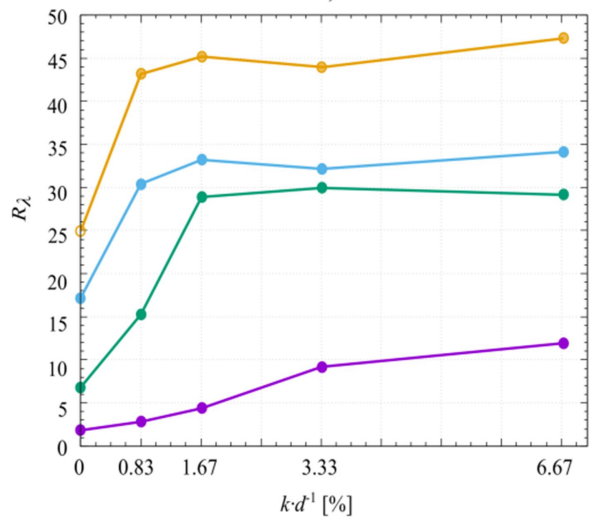

d)

Figure 5: Structure of turbulence in stream-wise direction depending on the relative roughness $k \cdot d^{-1}$ and reference velocity $U$. Purple, light blue, green and orange colors correspond to the different reference velocities $5,10,15$, and $20 \mathrm{~m} \cdot \mathrm{s}^{-1}$, respectively. While the graphs a), b), c) and d) show the distributions of the dissipation rate, dissipation microscale, Taylor microscale and Reynolds numbers based on the Taylor microscale, respectively. 
Finally, according to formula 5, we estimated the distribution of the Reynolds numbers based on the Taylor microscale depending on surface roughness and flow velocity (Fig. 4(d)). In general, the trends of the obtained curves are similar to the Taylor microscale distributions. As we can see, besides the flow velocity, the value of $R_{\lambda}$ also significantly depends on surface roughness. What is especially noticeable at low velocity. Thus, when the surface roughness increases to $k \cdot d^{-1} \sim 6.67 \%$, at $5 \mathrm{~m} \cdot \mathrm{s}^{-1}$ and $10 \mathrm{~m} \cdot \mathrm{s}^{-1}$, the $R_{\lambda}$ increase by five and three times, respectively.

\section{Conclusions}

The roughness effect of the circular cylinder on the Strouhal number was investigated. For this purpose, were used 55P14 and 55P15 hot-wire probes. The experimental value of Reynolds numbers based on the cylinder diameter was $5 \times 10^{3}<\operatorname{Re}_{\mathrm{d}}<2 \times 10^{4}$. Moreover, analyzed on the obtained data, we found that with increasing relative roughness up to $6.67 \%$ compared to a smooth cylinder, the Strouhal number decreased by $4.5 \%$. Meanwhile, the analysis of the turbulence structure showed that in the whole range of experimental velocities, the dissipation rate decreases by $13-16 \%$. While the dissipation scales increase approximately by $4 \%$. In addition, the spectral distribution of the obtained signals showed that with increasing roughness, regardless of the velocity, the vortex-shedding frequency decreases by $16.5 \%$.

\section{Acknowledgement}

This work was supported by the project of Technology Agency of the Czech Republic TACR No. TH02020057 "Program Epsilon" nd ERDF under project "Research Cooperation for Higher Efficiency and Reliability of Blade Machines (LoStr)” No. CZ.02.1.01 /0.0/0.0/16_026/0008389.

\section{References}

[1] Achenbach, E.: The effect of surface roughness on the heat transfer from a circular cylinder to the cross flow of air. International Journal of Heat and Mass Transfer, vol. 20, no. 4. (1977) pp. 359369.

[2] Zdravkovich, M.: The effects of interference between circular cylinders in cross flow. Journal of Fluids and Structures, vol. 1, (1987) pp. 239-261.

[3] Adachi, T. et al.: Study of the Universal Strouhal Number over the Wide Reynolds Number Flow Range. Effect of Surface Roughness. JSME International Journal Series B., vol. 39. (1996) pp. 335342.

[4] Kadivar, M. and Tormey, D. and Gerard McGranaghan, G.: A review on turbulent flow over rough surfaces: Fundamentals and theories. International Journal of Thermofluids, vol. 10, (2021) pp. 134.

[5] Hagen, G.: Uber den einfluss der temperatur auf die bewegung des wassers in rohren (on the influence of temperature on the movement of water in pipes), Math. Abh. Akad. Wiss, Berlin (1854).

[6] Darcy, H.: Recherches Experimentales Relatives au Mouvement de l'eau dans les Tuyaux (Experimental Research Relating to the Movement of Water in Pipes), Mallet-Bachelier, Paris, (1857).

[7] Schlichting, H.: Experimentelle untersuchungen zum rauhigkeitsproblem (experimental investigation of the problem of surface roughness), Arch. Appl. Mech. vol. 7 (1936) pp.15-34.

[8] Tsutomu, A: Effects of surface roughness on the universal Strouhal number over the wide Reynolds number range. Journal of Wind Engineering and Industrial Aerodynamics. vol. 69, (1997) pp. 399412.

[9] Sun, C. et al.: Effect of surface roughness heights on circular cylinder wakes, $22^{\text {nd }}$ Australasian Fluid Mechanics Conference, (2020).

[10] Achenbach, E.: Influence of surface roughness on the cross-flow around a circular cylinder. Journal of Fluid Mechanics, 46(2), (1971) pp. 321-335.

[11] Güven, O., Farell, C. and Patel, V.: Surface roughness effects on the mean flow past circular cylinders. Journal of Fluid Mechanics, vol (98), no.4, (1980) pp.673-701.

[12] Yanovych, V., Duda, D., Uruba, V. et al.: Anisotropy of turbulent flow behind an asymmetric airfoil. SN Appl. Sci., vol. 3, 885 (2021). 
[13] Barenblatt, G. and Goldenfeld, N.: Does Fully-Developed Turbulence Exist? Reynolds Number Independence versus Asymptotic Covariance. Physics of Fluids, vol (7), no. 12, (1995) pp. 1-13.

[14] Bourgoin, M. et al.: Investigation of the small-scale statistics of turbulence in the modane S1MA wind tunnel. CEAS Aeronautical Journal, vol. 9, (2018) pp. 269-281.

[15] Yanovych V, Duda D, Uruba V.: Structure turbulent flow behind a square cylinder with an angle of incidence, European Journal of Mechanics - B/Fluids, vol 85, (2021) pp. 110-123. 